\title{
Field emission from as grown and nitrogen incorporated tetrahedral amorphous carbon/silicon heterojunctions grown using a pulsed filtered cathodic vacuum arc technique
}

\author{
O. S. Panwar ${ }^{\text {a) }}$ \\ Plasma Processed Materials Group, National Physical Laboratory, Dr. K.S. Krishnan Road, \\ New Delhi-110012, India \\ Nalin Rupesinghe and G. A. J. Amaratunga \\ Department of Engineering, University of Cambridge, Trumpington Street, Cambridge CB2 1PZ, England
}

(Received 21 August 2007; accepted 29 January 2008; published 28 March 2008)

\begin{abstract}
This article reports the field emission measurements on as grown tetrahedral amorphous carbon (ta-C) and nitrogen incorporated tetrahedral amorphous carbon (ta-C: N) films grown using a pulsed filtered cathodic vacuum arc technique. The effect of varying thickness on field emission in the as grown ta-C films and the effect of varying nitrogen content in ta-C: $\mathrm{N}$ films have also been studied. The values of threshold field of emission $\left(E_{\text {turn on }}\right)$ increase with decrease of thickness in the as grown ta-C films. Nitrogen incorporation up to 5.2 at. \% in ta-C films decreases the value of $E_{\text {turn on }}$ from 9.9 to $5.1 \mathrm{~V} / \mu \mathrm{m}$ and thereafter it starts increasing again. To understand the mechanism of electron emission, a realistic energy band diagram of ta-C:N/n $n^{++} \mathrm{Si}$ heterojunction has been proposed from the experimentally measured valence and conduction band offsets, using in situ $\mathrm{x}$-ray photoelectron spectroscopy and optical spectroscopy data already published in DRM 9 (2000) 1148. The data are explained using the Fowler and Nordheim theory. The field emission results obtained reveal that there exists a barrier to emission and the main barrier is at the front surface and this is related to the conduction band offset of the ta-C:N/n $n^{++}$Si heterojunction. (C) 2008 American Vacuum Society. [DOI: 10.1116/1.2884762]
\end{abstract}

\section{INTRODUCTION}

Field emission from carbon based thin film materials is a topic of increasing research interest activity due to the requirements of cold cathodes to be used in a new generation of field emission display devices and vacuum electronic devices. ${ }^{1-4}$ Initial interest was stimulated by the prospect of electron emission at very low electric fields due to the low electron affinity of the carbon in the diamond form when terminated with hydrogen: the diamond surface, in fact, has a negative electron affinity (NEA). Various carbon based cold cathodes including diamond, ${ }^{5}$ nanodiamond, ${ }^{6}$ diamondlike carbon (DLC) and tetrahedral amorphous carbon, ${ }^{7,8}$ carbon nanotubes, ${ }^{9,10}$ and nanoclustered carbon ${ }^{11-14}$ have all been shown to emit electrons at reasonably low electric fields. The ease of emission from widely different carbon materials suggests that NEA is not a prerequisite and may be a bonus feature. However, the exact mechanism of field emission is yet to be fully understood. ${ }^{15}$ Amorphous forms of carbon are attractive as cold cathodes because they can be deposited at room temperatures, on glass substrates over large areas which are the preferred choice of cost effective, large area flat panel displays since other films involve high temperature processes. The tetrahedral amorphous carbon (ta-C) contains no hydrogen and has the remarkable properties of high density and high hardness similar to diamond and contains $80 \%-$ $85 \% s p^{3}$ contents. These films have smooth surface and are potentially useful for electronic applications. The smooth-

${ }^{a)}$ Electronic mail: ospanwar@mail.nplindia.ernet.in ness in these films may be attributed to the $s p^{2}$ layer on the surface. The field emission characteristics of the amorphous carbon are in many ways better suited for display applications than diamond. Several models have been suggested in the literature for electron emission of $a-\mathrm{C}$ thin films. ${ }^{7,15-17} \mathrm{~A}$ space charge induced band bending and hot electron transport model was proposed by Amaratunga and Silva ${ }^{7}$ to explain the low field electron emission from nitrogen doped hydrogenated amorphous carbon $(a-\mathrm{C}: \mathrm{H}: \mathrm{N})$ thin films. In this model, they suggest that electrons from the back contact enter the $a$-C film (via tunneling or thermal excitation) under the influence of an internal applied electric field and gain enough energy during transport in the depleted $a-\mathrm{C}: \mathrm{H}: \mathrm{N}$ space charge region to surmount the relatively small barrier $(\sim 2-3 \mathrm{eV})$ for emission into vacuum. Forest et al. ${ }^{17}$ have argued that their observation of a minimum threshold field for electron emission at an optimal film thickness can be best explained by the space charge induced band bending model. Zhao et al. ${ }^{18}$ have, however, reported thickness independent electron field emission from ta-C films grown by filtered cathodic vacuum arc (FCVA) technique. In contrast, Hart et al. ${ }^{16}$ found no correlation between the threshold voltage and the work function of the back contact for field emission from ta-C films deposited on various metals. Robertson ${ }^{15}$ has argued using photoemission data from $a-\mathrm{C}: \mathrm{H}$ (Ref. 19) that the primary barrier lies at the front surface of the films. He proposed that electron emission from $a$-C films occurs due to large variation in the electron affinity in $\mathrm{C}-\mathrm{H}$ terminated and unterminated surface regions causing the electric field 
from the anode to be concentrated on the unhydrogenated areas. The focusing of the electric field on the negative charges results in downward band bending allowing electron emission via the Fowler-Nordheim process ${ }^{20}$ without exceeding the material breakdown field.

In this article, we report the electron field emission from the as grown (undoped) and nitrogen incorporated tetrahedral amorphous carbon (ta-C and ta-C: N) films grown using a pulsed FCVA technique on $n^{++}$silicon substrates. The effect of varying film thickness on electron field emission in the as grown ta- $\mathrm{C}$ and the effect of varying nitrogen content in ta-C: $\mathrm{N}$ films have been studied. The valence and conduction band offsets, measured for as grown ta- $\mathrm{C}$ and nitrogen incorporated ta-C, with varying $\mathrm{N}$ content using in situ $\mathrm{x}$-ray photoelectron spectroscopy (XPS) and optical spectroscopy published earlier ${ }^{21}$ have been used to propose a realistic energy band diagram of ta-C:N $/ n^{++} \mathrm{Si}$ heterojunction. Finally, the field emission properties of the as grown and nitrogen incorporated ta-C films are correlated with the band offset data.

\section{EXPERIMENTAL DETAILS}

\section{A. Details of the deposition of ta-C and ta-C: $\mathrm{N}$ films and FCVA system}

The deposition of the as grown ta-C and ta-C: $\mathrm{N}$ films were carried out on well cleaned highly doped $\langle 100\rangle n^{++}$silicon wafer and quartz substrates kept at room temperature using a pulsed FCVA process, where the carbon plasma is obtained by contacting the graphite cathode of $99.999 \%$ purity with a retractable graphite anode of the same purity. The FCVA system consists of a water cooled cathode and anode, $90^{\circ}$ bent magnetic filter for the removal of macroparticles, the substrate holder in a deposition chamber and turbo pump backed by a rotary pump, and the arc supply capable of delivering 130 A current. The carbon plasma is then transported around a $90^{\circ}$ bent filter to remove the macroparticles generated by the arc. The axial magnetic field produced is of $\sim 25 \mathrm{mT}$ which guides the ions to follow the same curved path. The incident ion energy is kept fixed at $\sim 22 \mathrm{eV}$ which was measured at the substrate position using retarding potential analyzer with a Faraday cup. A base pressure of better than $10^{-7}$ Torr was achieved which rose to $10^{-6}$ Torr during striking of the arc for the deposition of ta-C films. The arc was run at a current of $130 \mathrm{~A}$ and arc on voltage of $20 \mathrm{~V}$ for a time period of $1 \mathrm{~s}$ and then cooled for $30 \mathrm{~s}$. After ten pulses, the system was cooled for $5 \mathrm{~min}$ and then the sequence repeated. The deposition rate achieved was $\sim 0.6 \AA / \mathrm{s}$. Nitrogen incorporation in the growing ta-C films was made by introducing high purity nitrogen gas (Matheson) into the FCVA system using mks mass flow meter and controller. The nitrogen partial pressure was varied from $10^{-5}$ to $10^{-3}$ Torr in order to vary the amount of nitrogen concentrations in ta-C: $\mathrm{N}$ films. The nitrogen $(\mathrm{N})$ content in the film was evaluated using electron dispersive $\mathrm{x}$-ray analysis study and the values evaluated are found to be consistent with those using XPS peak intensities of carbon and nitrogen by standard procedure ${ }^{21}$ at the same nitrogen partial pressure.

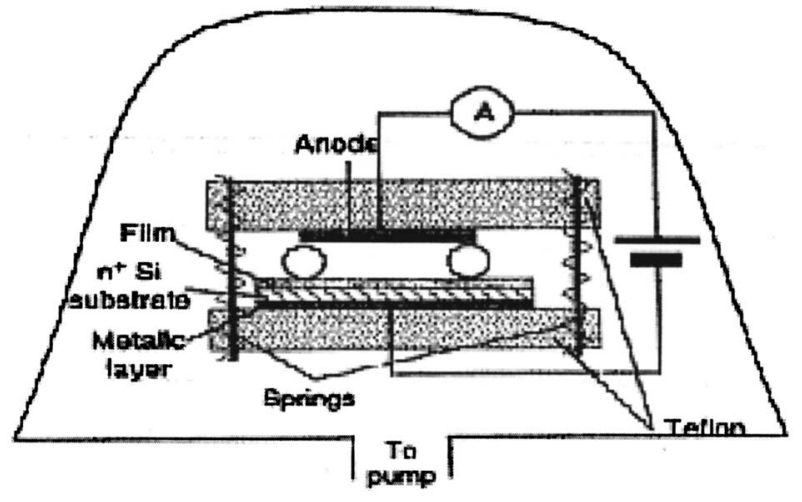

FIG. 1. Schematic representation of the field emission measurement.

The ta-C films grown using FCVA technique by Satyanarayana $e t a l .{ }^{8}$ were generally atomically smooth and root mean square roughness of the as-deposited ta-C films studied by atomic force microscopy was found to be a few angstroms $(0.06 \mathrm{~nm}$ or less). However, with very high $\mathrm{N}$ concentrations, there have been reports of nanoclustering varying from a few nanometers to $100 \mathrm{~nm}$ which is beyond the scope of this study. The thickness of these films was measured using a Dektak 3030 profilometer and also by Talystep thickness profiler (Rank-Taylor and Hobson).

\section{B. Details of the field emission measurement}

Field emission measurements were carried out in the parallel plate configuration for all the thin film samples with highly doped $n^{++}$silicon as the substrate in a vacuum $\sim 3$ $\times 10^{-7}$ Torr, created by a diffusion pump having a liquid nitrogen trap and backed by a rotary pump. An evaporated metal (Al) film, made in a vacuum $\sim 10^{-7}$ Torr, on the cleaned glass substrate was used as the anode plate. Unless otherwise stated all cathode samples were of $\sim 1 \mathrm{~cm}^{2}$ area. The metal area of the anode plate was smaller than the film on the silicon cathode (typically $0.25 \mathrm{~cm}^{2}$ ). The anode plate was separated from the cathode using high quality optical grade quartz fiber spacer of 50-80 $\mu \mathrm{m}$. The schematic of the field emission measurement is shown in Fig. 1. All the measurements were obtained using a Keithley source measure/ unit under computer control with in the LAB VIEW software suite. An inductive filter was used to prevent rapid current transient (microarcs) on the large area thin film cathode. Without such filtering, microarcs can cause destruction of the cathode surface at discrete locations once the exponential rise of emitted current with rising local field associated with field emission commences. No cathode surface degradation due to arcing was seen by microscopy after field emission characterization. The experiment of field emission was also carried out on uncoated $\mathrm{Si}$ substrates and by reversing the polarity of anode-cathode terminals. No currents (less than $1 \mathrm{pA}$ ) were measured up to a field of $15-20 \mathrm{~V} / \mu \mathrm{m}$ when a silicon substrate was used. The instrument was sensitive down to $1 \mathrm{pA}$ current only. It may also be emphasized that no conditioning process was used for the field emission mea- 

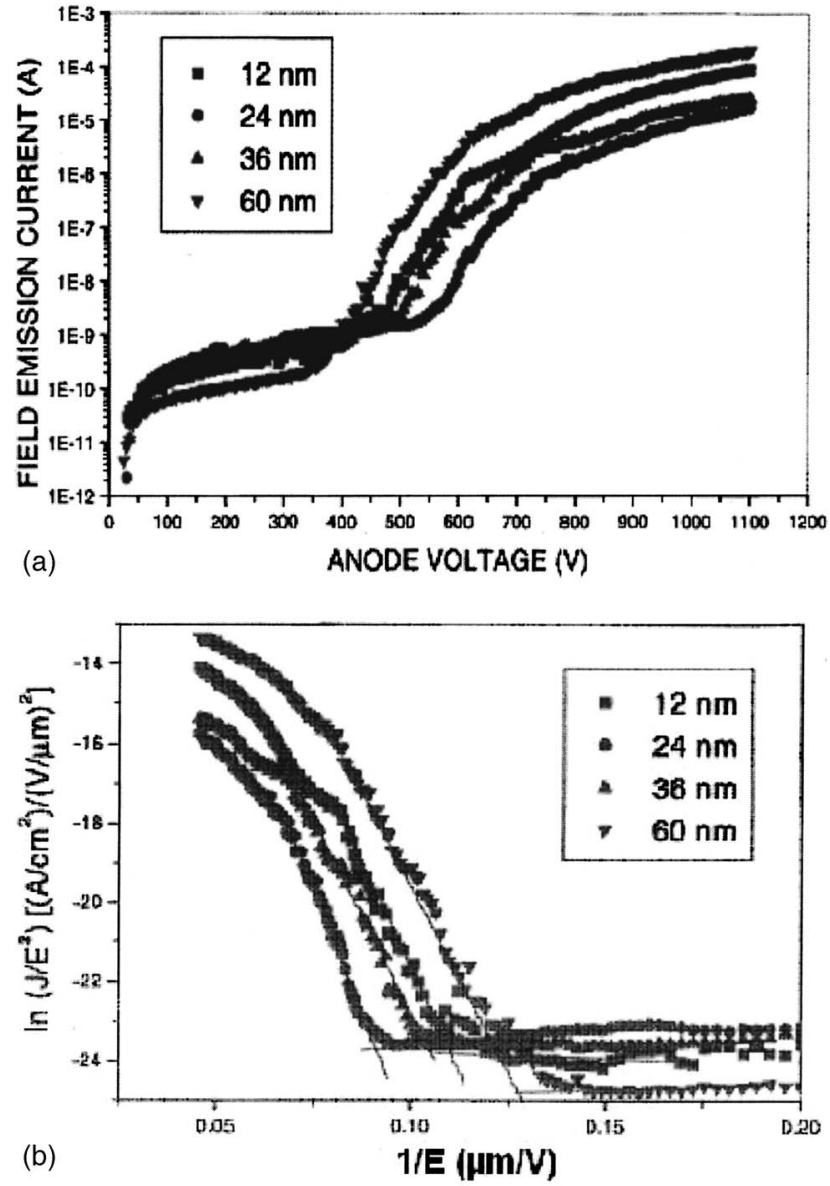

FIG. 2. (a) Variation of the field emission current (I) vs applied anode voltage $(V)$ for the as grown ta-C films using a pulsed FCVA technique with different thicknesses. (b) FN plot of the ta-C films with different thicknesses of the data shown.

surements on nitrogen doped ta-C films because the emission of our samples is found to be stable and repeatable. As grown (undoped) ta-C films, however, seem to require some conditioning process and the emission becomes stable and repeatable only after two to three runs.

\section{RESULTS}

\section{A. Field emission characteristics of thickness dependence of the as grown ta-C films}

The emission current $(I)$ versus anode voltage $(V)$ of ta-C films grown using a pulsed FCVA technique at different thicknesses varying from 12 to $60 \mathrm{~nm}$ are shown in Fig. 2(a). These characteristics are found to be repeatable and reproducible on the same sample, at different locations, and also on the sample prepared in different runs under identical conditions over periods of weeks in vacuum chamber. Exposure to atmosphere does not degrade the emission properties of the films. The current density is calculated by taking the anode area and the electric field is obtained by taking the entire applied voltage across the vacuum gap. Field emission involves a quantum mechanical process in which electrons tunnel out of the electrodes into vacuum when subjected to a very high electric field. It is a nonlinear process in which the emission current density $(J)$ versus electric field characteristics are usually described by the classical Fowler-Nordheim (FN) equation. ${ }^{20}$

$$
J=A(\gamma E)^{2} \exp \left(-B \phi^{3 / 2} / \gamma E\right),
$$

where $J$ is the current density in $\mathrm{A} / \mathrm{cm}^{2}$ at the point of emission, $V$ is the voltage applied across the vacuum gap $(d)$, $E(V / d)$ is the electric field in $\mathrm{V} / \mu \mathrm{m}$ across the vacuum gap, $\gamma$ is the local (microscopic) enhancement factor of the electric field at the point of emission, $\phi$ is the potential barrier in $\mathrm{eV}$ to electron emission. The terms $A$ and $B$ are constants with $A=1.4 \times 10^{-2} \mathrm{~A} \mathrm{~V}^{-2}$ and $B=6.8 \times 10^{9} \mathrm{~V} / \mathrm{m} \mathrm{eV}^{2 / 3}$, assuming that the effective mass of the field emitted electron is equal to that of an electron at rest in the metal and vacuum. The plots of $\ln \left(J / E^{2}\right)$ vs $1 / E$ for the data shown in Fig. 2(a) are depicted in Fig. 2(b). These come out to be straight lines which confirm that $J-E$ (or $I-V$ ) characteristics follow the FN relation. The slopes of these plots give the effective emission barriers $(\phi)$, if we assume an ideal plane emitter with a field enhancement factor $\gamma$ of 1 . The values of $\phi$ for the as grown ta-C and ta-C: $\mathrm{N}$ films grown, in this article, have been found to be very low (less than or equal to $0.1 \mathrm{eV}$ ). Similar values have also been reported by others. ${ }^{7,8}$ These values are obviously quite low, and the true barrier may be much larger. The value of the threshold field of emission $\left(E_{\text {turn on }}\right)$ is evaluated from the intersection point of the two straight lines in the FN curve. The values of $E_{\text {turn on }}$ together with the slope $m$ of FN curve and emission current density $(J)$ at $20 \mathrm{~V} / \mu \mathrm{m}$ at different thicknesses have been summarized in Table I. The values of $E_{\text {turn on }}$ and slope $m$ of FN curve are found to increase from 7.4 to $10.4 \mathrm{~V} / \mu \mathrm{m}$ and $1.63 \times 10^{8}$ to $2.24 \times 10^{8} \mathrm{~V} / \mathrm{m}$ respectively whereas that of emission current density $(J)$ are found to decrease from $6.4 \times 10^{-4}$ to $3.8 \times 10^{-5} \mathrm{~A} / \mathrm{cm}^{2}$ with

TABLE I. Electron emission properties of ta-C films with different thicknesses.

\begin{tabular}{|c|c|c|c|c|c|}
\hline \multirow[b]{2}{*}{ Serial No. } & \multirow[b]{2}{*}{ Properties } & \multicolumn{4}{|c|}{ Thickness (nm) } \\
\hline & & 12 & 24 & 36 & 60 \\
\hline 1 & $E_{\text {turn on }}(\mathrm{V} / \mu \mathrm{m})$ & 9.2 & 10.4 & 9.5 & 7.4 \\
\hline 2 & Slope $m(\mathrm{~V} / \mathrm{m})$ of $\mathrm{FN}$ curve & $2.28 \times 10^{8}$ & $2.24 \times 10^{8}$ & $1.85 \times 10^{8}$ & $1.63 \times 10^{8}$ \\
\hline 3 & $J\left(\mathrm{~A} / \mathrm{cm}^{2}\right)$ at $20 \mathrm{~V} / \mu \mathrm{m}$ & $2.4 \times 10^{-4}$ & $3.8 \times 10^{-5}$ & $7.2 \times 10^{-5}$ & $6.4 \times 10^{-4}$ \\
\hline 4 & $\gamma_{c}$ & 289.36 & 295.07 & 356.52 & 405.80 \\
\hline 5 & $\gamma_{f}$ & 143.34 & 146.28 & 176.74 & 201.17 \\
\hline 6 & $\gamma_{v}$ & 318.44 & 324.73 & 392.35 & 446.59 \\
\hline 7 & $\gamma_{\Delta E_{c}}$ & 39.19 & 39.97 & 48.29 & 54.96 \\
\hline
\end{tabular}


the decrease of film thickness from 60 to $24 \mathrm{~nm}$. However, $12 \mathrm{~nm}$ thick ta-C film shows reversal of trend with $E_{\text {turn on }}$ $=9.2 \mathrm{~V} / \mu \mathrm{m}$ and $J=2.4 \times 10^{-4} \mathrm{~A} / \mathrm{cm}^{2}$ having the value of slope $m=2.28 \times 10^{8} \mathrm{~V} / \mathrm{m}$. A strong relationship of electron field emission with film thickness has been found by Forest et $a l .{ }^{17}$ and Cheah et al. ${ }^{22}$ According to these authors, the values of $E_{\text {turn on }}$ are found to decrease up to certain thickness of $30 \mathrm{~nm}$ in ta-C: $\mathrm{N}$ films grown by FCVA technique and $60 \mathrm{~nm}$ in $a-\mathrm{C}: \mathrm{H}$ films grown by rf plasma-enhanced chemical-vapor deposition (PECVD) technique and thereafter the values of $E_{\text {turn on }}$ are found to increase steadily. There is a minimum turning point in the values of $E_{\text {turn on }}$ with film thickness which indicates that there is a window for optimizing field emission properties of these films. However, opposite experimental results of thickness independent field emission from ta-C films grown by filtered arc deposition have been reported by Zhao et al. ${ }^{18}$ It may also be mentioned that the trend observed in the present investigation on thickness dependence of field emission of ta-C films is found to be consistent with that observed by Forest et al. ${ }^{17}$ and Cheah et $a{ }^{22}$ in ta-C films in the limited range of 24-60 nm studied here. We, however, observed maxima in $E_{\text {turn on }}$ of $24 \mathrm{~nm}$ thick ta-C films studied in the range of $12-60 \mathrm{~nm}$ in the present study, whereas they have observed minima at $30 \mathrm{~nm}$ thickness of ta-C films studied in the range of 10-90 nm.

\section{B. Field emission characteristics of ta-C: $\mathbf{N}$ films}

The emission current $(I)$ versus applied voltage $(V)$ of ta-C: $\mathrm{N}$ films (30 $\mathrm{nm}$ thick) grown with varying nitrogen $(\mathrm{N})$ contents using a pulsed FCVA technique are shown in Fig. 3(a). The plots of $\ln \left(J / E^{2}\right)$ vs $1 / E$ for the data shown in Fig. 3(a) are depicted in Fig. 3(b). These come out to be straight lines which confirm that $J-E$ (or $I-V$ ) characteristics follow the FN relation. The values of $E_{\text {turn on }}$ together with the slope $m$ of the FN curve and the emission current density $(J)$ at $12 \mathrm{~V} / \mu \mathrm{m}$ of ta-C: $\mathrm{N}$ films with varying $\mathrm{N}$ contents have been summarized in Table II. The value of $E_{\text {turn on }}$ is found to be $9.9 \mathrm{~V} / \mu \mathrm{m}$ for as grown ta-C film (30 nm thick) and it decreases with the increase of $\mathrm{N}$ content up to 5.2 at. $\%$ in ta-C: $\mathrm{N}$ films and thereafter it starts increasing again and it becomes $6.6 \mathrm{~V} / \mu \mathrm{m}$ at 11.4 at. $\%$ of $\mathrm{N}$ content and $9.4 \mathrm{~V} / \mu \mathrm{m}$ at 16.6 at. $\%$ of $\mathrm{N}$ content. The value of the slope $m$, evaluated from the $\mathrm{FN}$ curve is found to be 1.97 $\times 10^{8} \mathrm{~V} / \mathrm{m}$ and it shows decreasing trend up to 3.6 at. $\% \mathrm{~N}$ content having the value of slope $m=1.11 \times 10^{8} \mathrm{~V} / \mathrm{m}$ and thereafter it starts increasing again and becomes 2.81 $\times 10^{8} \mathrm{~V} / \mathrm{m}$. The value of $J$ at $12 \mathrm{~V} / \mu \mathrm{m}$ for the as grown ta-C film is $4.8 \times 10^{-7} \mathrm{~A} / \mathrm{cm}^{2}$ and it increases with the incorporation of $\mathrm{N}$ content up to 2.9 at. $\%$ and becomes 2.6 $\times 10^{-4} \mathrm{~A} / \mathrm{cm}^{2}$ and thereafter it decreases again with the further increase of $\mathrm{N}$ content. Thus, low levels of nitrogen incorporation are found to increase emission significantly but higher levels of nitrogenation reduce it since they induce graphitization. Satyanarayana et $a l^{8}$ have also studied field emission from ta-C films grown by FCVA technique and they have reported that the values of $E_{\text {turn on }}$ decrease with the
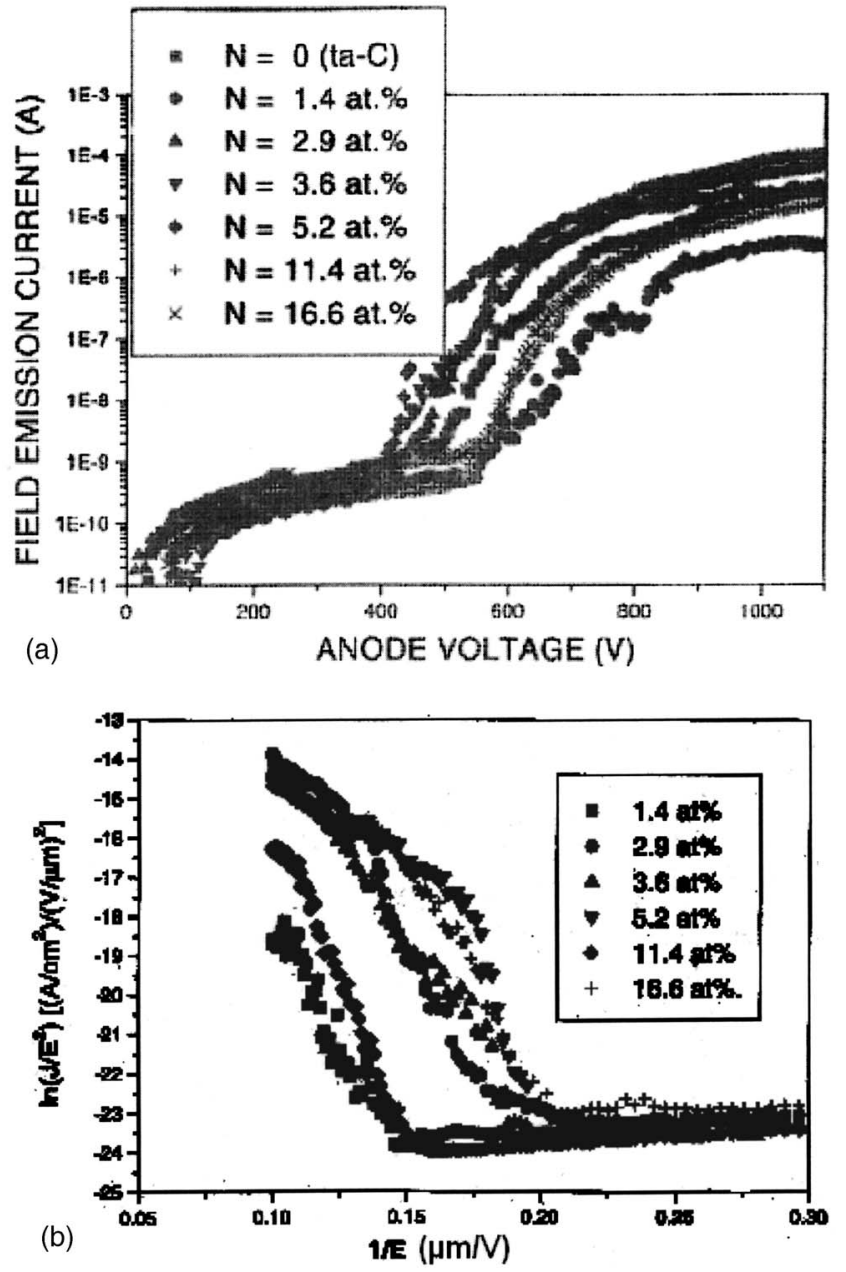

FIG. 3. (a) Variation of the field emission current (I) vs applied anode voltage $(V)$ for ta-C: $\mathrm{N}$ films with different $\mathrm{N}$ contents grown using a pulsed FCVA technique. (b) FN plot for the ta-C: $\mathrm{N}$ films with different $\mathrm{N}$ contents of the data shown.

increase of $s p^{3}$ content as well as with low levels of nitrogen content. They reported the minimum values of $E_{\text {turn on }}$ $=10-12 \mathrm{~V} / \mu \mathrm{m}$ for an optimum ion energy of $80-100 \mathrm{eV}$ in ta-C films and optimally nitrogen doped $(0.4$ at. $\% \mathrm{~N})$ films recorded $E_{\text {turn on }}$ values as low as $3-5 \mathrm{~V} / \mu \mathrm{m}$. Amaratunga and Silva ${ }^{7}$ have also studied nitrogen containing $a-\mathrm{C}: \mathrm{H}$ films grown by rf PECVD technique with magnetic confinement and they reported that the onset emission field decreases with the increase of $\mathrm{N}$ content and onset emission field as low as $4 \mathrm{~V} / \mu \mathrm{m}$ has been obtained in $a-\mathrm{C}: \mathrm{H}$ films having 15 at. $\%$ $\mathrm{N}$ content. The value of $E_{\text {turn on }}=9.9 \mathrm{~V} / \mu \mathrm{m}$ for as grown ta-C films grown using a pulsed FCVA technique at $\sim 22 \mathrm{eV}$ ion energy is found to be less than the value of $E_{\text {turn on }}$ $=22 \mathrm{~V} / \mu \mathrm{m}$ in ta-C films grown at approximately the same ion energy and less than $10-12 \mathrm{~V} / \mu \mathrm{m}$ reported, at the optimum ion energy of $80-100 \mathrm{eV}$ of ta-C films grown by direct FCVA technique by Satyanarayana et al. ${ }^{8}$ Thus, as grown ta-C and ta-C: $\mathrm{N}$ films grown using a pulsed FCVA technique in the present investigation which show less values of $E_{\text {turn on }}$ and larger emission current density than the values reported by Satyanarayana et $a .^{8}$ in the films grown using direct 
TABLE II. Electron emission properties of ta-C: $\mathrm{N}$ films with different $\mathrm{N}$ contents.

\begin{tabular}{|c|c|c|c|c|c|c|c|c|}
\hline \multirow{2}{*}{$\begin{array}{c}\text { Serial } \\
\text { No. }\end{array}$} & \multirow[b]{2}{*}{ Properties } & \multicolumn{7}{|c|}{$\mathrm{N}$ content (at \%) } \\
\hline & & 0 & 1.4 & 2.9 & 3.6 & 5.2 & 11.6 & 16.6 \\
\hline 1 & $E_{\text {turn on }}(\mathrm{V} / \mu \mathrm{m})$ & 9.9 & 6.8 & 5.7 & 5.3 & 5.1 & 6.6 & 9.4 \\
\hline 2 & $J\left(\mathrm{~A} / \mathrm{cm}^{2}\right)$ at $12 \mathrm{~V} / \mu \mathrm{m}$ & $4.8 \times 10^{-7}$ & $1.0 \times 10^{-5}$ & $2.6 \times 10^{-4}$ & $2.1 \times 10^{-4}$ & $1.2 \times 10^{-4}$ & $3.3 \times 10^{-5}$ & $2.8 \times 10^{-6}$ \\
\hline 3 & Slope $m(\mathrm{~V} / \mathrm{m})$ & $1.97 \times 10^{8}$ & $1.26 \times 10^{8}$ & $1.53 \times 10^{8}$ & $1.11 \times 10^{8}$ & $2.10 \times 10^{8}$ & $1.69 \times 10^{8}$ & $2.81 \times 10^{8}$ \\
\hline 4 & $\chi(\mathrm{eV})$ & 2.85 & 2.55 & 2.65 & 2.75 & 3.65 & 3.35 & 3.15 \\
\hline 5 & $\phi(\mathrm{eV})$ & 4.55 & 4.15 & 4.15 & 4.15 & 4.25 & 3.95 & 4.05 \\
\hline 6 & $\phi\left(\phi+\Delta E_{f}\right)(\mathrm{eV})$ & 4.85 & 4.55 & 4.65 & 4.75 & 5.15 & 4.65 & 4.45 \\
\hline 7 & $\Delta E_{c}(\mathrm{eV})$ & 1.2 & 1.5 & 1.4 & 1.3 & 0.4 & 0.7 & 0.9 \\
\hline 8 & $\gamma_{c}$ & 166.08 & 219.76 & 191.73 & 279.37 & 225.80 & 245.98 & 135.29 \\
\hline 9 & $\gamma_{f}$ & 335.01 & 456.26 & 375.74 & 517.91 & 283.71 & 314.95 & 197.24 \\
\hline 10 & $\gamma_{v}$ & 368.68 & 523.79 & 445.65 & 634.20 & 378.44 & 402.27 & 227.17 \\
\hline 11 & $\gamma_{\Delta E_{c}}$ & 45.37 & 99.15 & 73.62 & 90.80 & 8.19 & 14.18 & 14.17 \\
\hline 12 & $\Delta E_{v}^{c}(\mathrm{eV})$ & 0.3 & 0.6 & 0.5 & 0.4 & 0 & 0.5 & 0.7 \\
\hline 13 & $\Delta E_{F}(\mathrm{eV})$ & 0.3 & 0.4 & 0.5 & 0.6 & 0.9 & 0.7 & 0.4 \\
\hline 14 & $E_{g}(\mathrm{eV})$ & 2.0 & 2.0 & 2.0 & 2.0 & 1.5 & 1.3 & 1.3 \\
\hline
\end{tabular}

FCVA technique, are found to be superior. Park et al., ${ }^{23}$ however, reported that the room temperature conductivity as well as the emission current in nitrogen doped ta-C films decrease with the increase of low amount of nitrogen content and thereafter it increases at higher amount of $\mathrm{N}$ content even though the conductivity and emission minimum occur at different $\mathrm{N}$ contents. The emission current, in the present investigations, is found to increase with the incorporation of $\mathrm{N}$ content up to 2.9 at. \% having a maxima and thereafter it decreases which is contrary to the observation reported by Park et al. ${ }^{23}$ Weber et $a l .{ }^{24}$ have reported the onset emission field as low as $3.2 \mathrm{~V} / \mu \mathrm{m}$ in a-C: $\mathrm{N}$ films with 0.6 at. $\% \mathrm{~N}$ content grown by sputtering a graphite employing an ECR plasma as argon ion source. They achieved current densities up to $0.6 \mathrm{~mA} / \mathrm{cm}^{2}$ at an electric field of $5.8 \mathrm{~V} / \mu \mathrm{m}$ which is, however, larger than the maximum values achieved in the present study. The values of $E_{\text {turn on }}$ reported here are found to be lower than the values obtained by Park et al. ${ }^{33}$ and Chuang et $a .^{25}$ and significantly lower than those of undoped $a-\mathrm{C}: \mathrm{H}$ films by Lee et $a{ }^{26}{ }^{26}$ and lower than $20-60 \mathrm{~V} / \mu \mathrm{m}$ for the crater free emission from ta-C deposited by laser ablation. ${ }^{27}$

\section{CONSTRUCTION OF THE ENERGY BAND DIAGRAM OF ta-C: $\mathrm{N} / \boldsymbol{n}^{++} \mathrm{Si}$ HETEROJUNCTION}

\section{A. XPS study}

The understanding of the emission mechanism has been hindered by the lack of any measured experimental data on the band offsets between ta-C and Si. The only data available are that obtained for hydrogenated amorphous carbon $(a-\mathrm{C}: \mathrm{H})$ on $p$-type $\mathrm{Si}^{19,28}$ All photoemission measurements were carried out with monochromatic $\mathrm{Al} K \alpha(1486.6 \mathrm{eV}) \mathrm{x}$ rays using the same pulsed FCVA deposition system attached directly to an ultrahigh vacuum XPS chamber via a load lock chamber. $^{21}$

The band diagram for ta-C: $\mathrm{N}$ on $n^{++} \mathrm{Si}$ from the valence band offsets for the as grown (undoped) ta-C and nitrogen doped ta-C, with six different amounts of $\mathrm{N}$ concentrations have been studied earlier. ${ }^{21}$ The realistic energy band diagram drawn from the calculated band diagram, ${ }^{21}$ is shown in Fig. 4 using the evaluated values of electron affinity $(\chi)$ and work function $(\phi)$ described in Sec. IV B together with the band offsets. The values of conduction band offset $\left(\Delta E_{c}\right)$, valence band offset $\left(\Delta E_{v}\right), \Delta E_{F}\left(E_{F}-E_{v}\right)$ and optical band gap $\left(E_{g}\right)$, shown in Fig. 4 of the as grown ta-C and ta-C: $\mathrm{N}$ at different $\mathrm{N}$ contents, have also been summarized in Table II. The band offsets reported in this article were between undoped and doped ta-C and $n^{++} \mathrm{Si}$. The $\mathrm{N}$ concentration in these films was determined using the $\mathrm{C} 1 s$ and $\mathrm{N} 1 s$ peak intensities and the sensitivity values of $\mathrm{N}(0.38)$ and $\mathrm{C}$ $(0.205)$ using the standard procedure. Salient points from the earlier study ${ }^{21}$ are reproduced here for the continuity purpose in this section. It is evident from the figure that the valence band edge of as deposited ta-C film lies $0.3 \mathrm{eV}$ below the Fermi level $\left(E_{F}\right)$, comparable to other reports, confirming its $p$-type nature. ${ }^{29,30}$ In contrast, the value of $\Delta E_{c}$ for ta-C film on $n^{++} \mathrm{Si}$ is significantly larger having value $1.2 \mathrm{eV}$. For nitrogen doped ta-C, several observations are derived from Fig. $4 .{ }^{21}$ Taking low $\mathrm{N}$ content of 1.4 at. \%, first an upward shift of $0.1 \mathrm{eV}$ is seen for the valence band of the substrate. Second, the value of $\Delta E_{v}$ has increased from $0.3 \mathrm{eV}$ for as deposited ta-C films to $0.6 \mathrm{eV}$ for ta-C: $\mathrm{N}$ at 1.4 at. $\% \mathrm{~N}$ content. The $E_{F}$ is observed to have shifted from 0.3 to $0.4 \mathrm{eV}$ toward the midgap. The shift of the $E_{F}$ from the conduction band suggests that some of the nitrogen acts as an electron donor in ta-C films. At the interface, nitrogen in ta-C seems to be incorporated to passivate the traps, thereby reducing the electron transfer from the $n^{++} \mathrm{Si}$ to ta-C seen as a reduction in upward band bending in $n^{++} \mathrm{Si}$ $(0.1 \mathrm{eV})$. Further for ta-C: $\mathrm{N}$ with 2.9 and 3.6 at. \% [Figs. 4(c) and 4(d)] the value of $\Delta E_{v}$ seems to decrease with the movement of the $E_{F}$ toward the midgap, away from the valence band. It should be noted that the shift in the measured valence band, as $\mathrm{N}$ content is increased from 1.4 to 5.2 at. \% is the same as the relative shift in the $E_{F}$. The value of $E_{g}$ remains the same at $2.0 \mathrm{eV}$ up to 3.6 at. $\% \mathrm{~N}$ content. With 5.2 at. $\% \mathrm{~N}$ content, the value of $E_{g}$ reduces to $1.5 \mathrm{eV}$ but the 


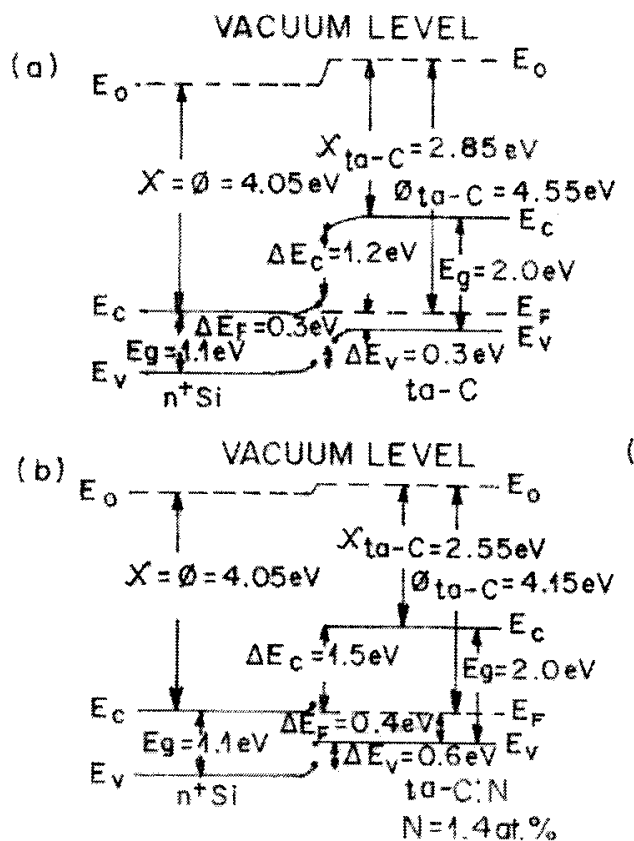

(d)
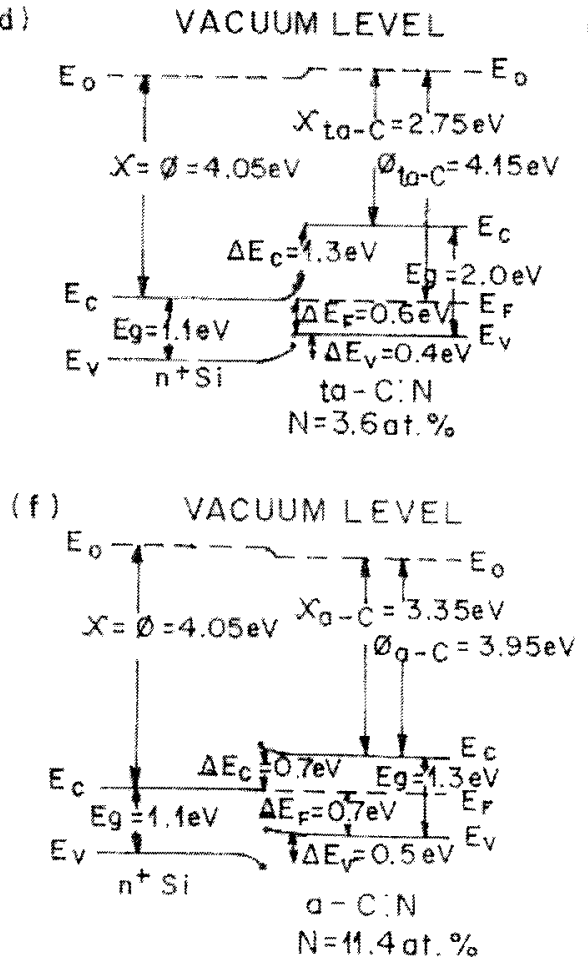

(c) VACUUM LEVEL

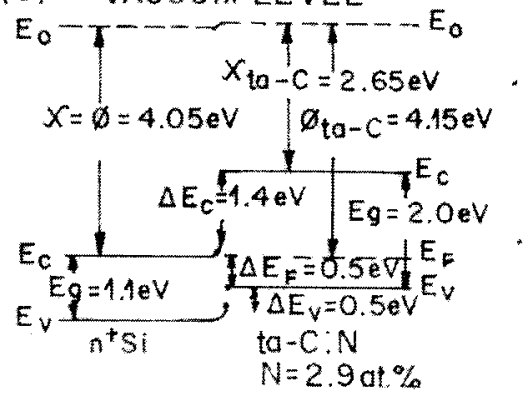

(e) VACUUM LEVEL
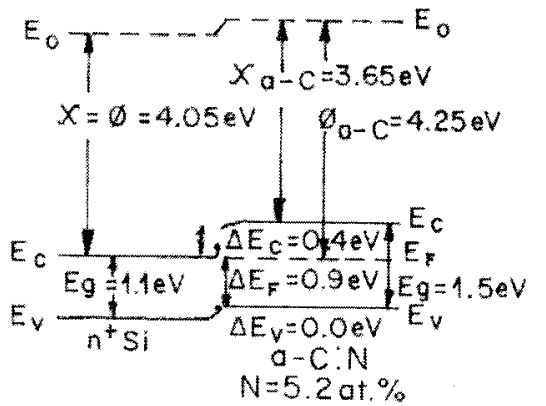

(g) VACUUM LEVEL

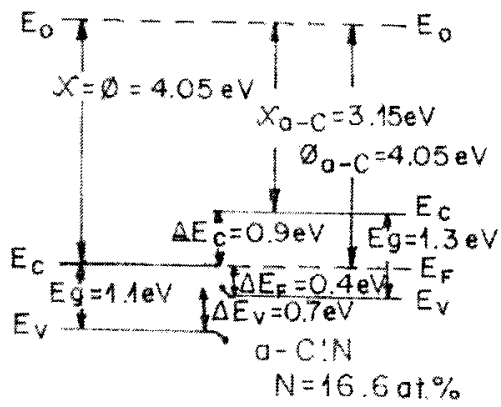

FIG. 4. Realistic energy band diagram of (a) as-deposited ta-C on $n^{++} \mathrm{Si}$ and ta-C: $\mathrm{N}$ films on $n^{++} \mathrm{Si}$ with six different $\mathrm{N}$ contents: (b) 1.4 at. \%, (c) 2.9 at. $\%$, (d) 3.6 at. $\%$, (e) 5.2 at. $\%$, (f) 11.6 at. $\%$, and $(\mathrm{g}) 16.6$ at. $\%$. trend in the valence band shift is maintained and the value of $\Delta E_{v}$ becomes zero. Additionally, with $E_{F}$ now being located beyond midgap, a downward band bending in the ta-C: $\mathrm{N}$ of $0.1 \mathrm{eV}$ is observed. When the $\mathrm{N}$ content increased beyond 5.2 at. \% the value of $E_{g}$ is found to reduce to $1.3 \mathrm{eV}$ for 11.4 and 16.6 at. $\% \mathrm{~N}$ contents and that of $\Delta E_{v}$ increases and $E_{F}$ shifts back toward the valence band. The relationship between the relative shift in the $E_{F}$ and the measured $\Delta E_{v}$ no longer applies beyond $\mathrm{N}=5.2$ at. $\%$. The increase in the value of $\Delta E_{v}$ in conjunction with the $E_{F}$ moving close to the valence band may be associated with the collapse of the conduction band at $\mathrm{N}$ content above some threshold value higher than 5.2 at. \%. The closing of the $E_{g}$ in ta-C with increasing $\mathrm{N}$ content has been observed in other studies and is also correlated to the sharp decrease in $s p^{3}$ fraction at higher $\mathrm{N}$ concentrations. ${ }^{31-33}$ The calculated band diagrams for all ta-C: $\mathrm{N}$ films examined ${ }^{21}$ show significant conduction band offset indicating that there exists a back barrier to the electron emission. 


\section{B. Energy band diagram}

The energy band model of an ideal abrupt heterojunction without interface traps were proposed by Anderson ${ }^{34}$ based on the previous work of Shockley and also mentioned as the basic device model in the literature. ${ }^{35}$ The energy band diagram of two isolated semiconductors of $n^{++} \mathrm{Si}$ and ta-C: $\mathrm{N}$ is already published. ${ }^{21}$ The two semiconductors have different $E_{g}$, different $\phi$, and different $\chi$. The difference in the energy of the conduction band edges in the two semiconductors is represented by $\Delta E_{c}$ and that in the valence band as $\Delta E_{v}$. When a junction is formed between the two semiconductors, the following steps are used to evaluate the values of $\chi$ and $\phi$ of as grown ta-C and ta-C: $\mathrm{N}$ films at different $\mathrm{N}$ contents. The value of $\chi$ for $n^{++} \mathrm{Si}$ is known and taken as $4.05 \mathrm{eV}$ and it can also be taken as the value of $\phi$ for $n^{++}$Si since the $E_{F}$ of highly doped semiconductor may be almost touching the conduction band. We adopted the following four steps in order to calculate the values of $\chi$ and $\phi$ for ta-C: $\mathrm{N}$ films with different $\mathrm{N}$ contents.

1. In the first step, from the energy levels of two different semiconductors, we can write the following equations as

$$
\begin{aligned}
& E_{c_{1}}-E_{g_{1}}=E_{v_{1}}, \\
& E_{c_{2}}-E_{g_{2}}=E_{v_{2}},
\end{aligned}
$$

where $E_{c_{1}}, E_{v_{1}}$, and $E_{g_{1}}$ are the conduction band level, valence band level, and the optical band gap of the first semiconductor, i.e., $n^{++} \mathrm{Si}$ and $E_{c_{2}}, E_{v_{2}}$, and $E_{g_{2}}$ are the conduction band level, valence band level, and the optical band gap of the second semiconductor, i.e., ta-C: $\mathrm{N}$ films at fixed $\mathrm{N}$ content.

2. In the second step, by subtracting Eqs. (3) from Eq. (2), we obtained

$$
E_{c_{1}}-E_{c_{2}}+E_{g_{2}}-E_{g_{1}}=E_{v_{1}}-E_{v_{2}},
$$

By substituting the experimentally measured values of the $E_{g_{1}}$ and $E_{g_{2}}$ of $n^{++} \mathrm{Si}$ and ta-C: $\mathrm{N}$ respectively, and the $\Delta E_{v}$ $\left(E_{v_{1}}-E_{v_{2}}\right)$, we can evaluate the value of $E_{c_{1}}-E_{c_{2}}\left(\Delta E_{c}\right)$.

3. In the third step, since the $E_{F}$ must coincide on both sides in the equilibrium condition $\left(E_{F_{1}}=E_{F_{2}}\right)$ and the vacuum level is everywhere parallel to the band edges and is continuous, we can write

$$
\chi_{1}+E_{c_{1}}-E_{F_{1}}=\chi_{2}+E_{c_{2}}-E_{F_{2}},
$$

By rearranging Eq. (5), we can write as

$$
\chi_{1}-\chi_{2}=E_{c_{2}}-E_{c_{1}} .
$$

By substituting the value of $\chi_{1}=4.05 \mathrm{eV}$ for $n^{++} \mathrm{Si}$ in Eq. (6) and the value of $E_{c_{2}}-E_{c_{1}}$ evaluated from Eq. (4) at fixed $\mathrm{N}$ content, we can derive the value of $\chi_{2}$ of as grown ta-C and ta-C: $\mathrm{N}$ with the same $\mathrm{N}$ content.

4. In the fourth step, we derive the value $\phi_{2}$ of as grown ta-C and ta-C: $\mathrm{N}$ at fixed $\mathrm{N}$ content, using the following equation:

$$
\phi_{2}=\chi_{2}+E_{g_{2}}-\Delta E_{F}
$$

By substituting the value of $\chi_{2}$ evaluated from Eq. (6) and $E_{g_{2}}$ and $\Delta E_{F}$ from published literature ${ }^{21}$ which are also shown in Fig. 4 and Table II, we can find out the value of $\phi_{2}$ of as grown ta- $\mathrm{C}$ and ta-C: $\mathrm{N}$ at a fixed $\mathrm{N}$ content. Thus, by adopting the above-mentioned four steps, we can derive the value of $\chi_{2}$ and $\phi_{2}$ of undoped ta-C and nitrogen doped ta-C: $\mathrm{N}$ at all the six $\mathrm{N}$ concentrations. The values of $\chi, \phi$, and $\phi_{v}$ $\left(\phi+\Delta E_{F}\right)$, corresponding local field enhancement factor from the conduction band, Fermi level, valence band and conduction band offsets, respectively, as $\gamma_{c}, \gamma_{f}, \gamma_{v}, \gamma_{\Delta E_{c}}$ of the as grown ta-C and ta-C: $\mathrm{N}$ films at different $\mathrm{N}$ concentrations have been summarized in Table II together with the values of $E_{\text {turn on }}$, slope $m$ of FN curve, $J$ at $12 \mathrm{~V} / \mu \mathrm{m}, \Delta E_{c}$, $\Delta E_{v}, \Delta E_{F}$, and $E_{g}$. Effort has been made to understand the origin of emission mechanism whether it is taking place either from the $E_{c}$ or from the $E_{F}$ or from the $E_{v}$, etc. It is evident from Table II that only the values of $\Delta E_{c}$ are found to follow the same trend as that of $E_{\text {turn on }}$ with varying $\mathrm{N}$ content whereas the values of $\phi$ and $\phi_{v}$ do not seem to follow the same trend. The corresponding values of $\gamma_{\Delta E_{c}}$ are found to be in the range of 8-100 for the emission due to $\Delta E_{c}$. The values of $\gamma_{c}$ are found to lie in the range from 135 to 280 corresponding to the emission due to $E_{c}$. This leads us to believe that the emission is taking place from the conduction band and this is probably due to the conduction band offset. Three parameters are thought to be the primary variables influencing the electron emission for carbon thin films: the nature of the back contact, transport through the film and the surface properties. Hart et al. ${ }^{16}$ while studying field emission from ta-C films as a function of surface treatment and substrate material stated that the threshold field and current densities for undoped ta-C films are found to be significantly improved by the plasma treatments largely due to an increase in the emission site densities, while little dependence was found on the work function of the substrate. This reveals that the main barrier to emission is at the front surface rather than at the back contact.

\section{DISCUSSION}

The conduction mechanism in these films is governed by the thermionic emission and it follows the RichardsonDushman equation

$$
J=A_{\mathrm{RD}} T^{2} \exp (-e \chi / k T),
$$

where $A_{\mathrm{RD}}$ is the Richardson-Dushman constant and $\chi$ is the electron affinity. The characteristics also follow conduction band offset $\left(\Delta E_{c}\right)$ in ta-C:N/n $n^{++}$Si heterojunction. The plots of $\ln \left(J / A_{\mathrm{RD}} T^{2}\right)$ vs $\chi$ as well as $\Delta E_{c}$ at different electric fields are shown in Figs. 5(a) and 5(b) respectively and they come out to be the straight line with a constant value of $e / k T$ $\sim 39$. Logarithmic value of current $(\log I)$ versus logarithmic value of anode voltage $(\log V)$ plots for ta-C films with different thicknesses and of ta-C: $\mathrm{N}$ films with different $\mathrm{N}$ contents are plotted in Figs. 6(a) and 6(b), respectively. It is evident from Figs. 6(a) and 6(b) that $\log I$ vs $\log V$ characteristics, in general, comprise of three parts. 

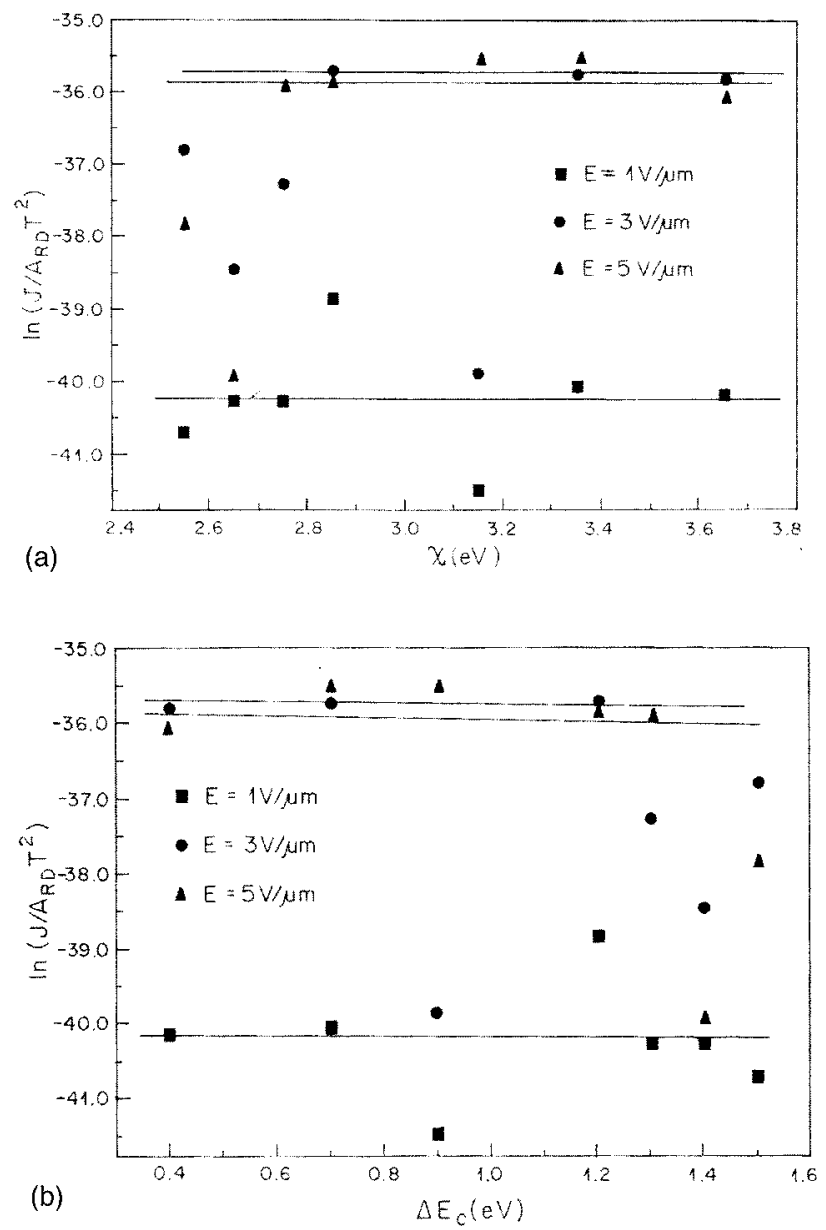

FIG. 5. (a) Typical variation of $\ln \left(J / A_{\mathrm{RD}} T^{2}\right)$ vs $\chi$ at different electric fields for ta-C film. (b) Typical variation of $\ln \left(J / A_{\mathrm{RD}} T^{2}\right)$ vs $\Delta E_{c}$ at different electric fields for ta-C film.

1. At lower field $E<E_{\text {turn on }}, \log I$ versus $\log V$ curve is having a unity slope indicating the Ohmic nature with emission current density $J<10^{-9} \mathrm{~A} / \mathrm{cm}^{2}$. This holds true for ta-C films with different thicknesses as well as for ta-C: $\mathrm{N}$ films at all the $\mathrm{N}$ concentrations.

2. At intermediate field, say $E<E_{\text {turn on }}<12-15 \mathrm{~V} / \mu \mathrm{m}$ for ta-C films with different thicknesses and for $E<E_{\text {turn on }}$ $<6-12 \mathrm{~V} / \mu \mathrm{m}$ for ta-C: $\mathrm{N}$ films with different $\mathrm{N}$ contents, there is an abrupt increase in the current and it follows the Fowler-Nordheim equation (linear region) in the FN curve. $\log I$ vs $\log V$ curves have much larger slope ranging from 18 to 24 for ta-C films which increases with decrease of film thickness and from 15 to 35 for ta-C: $\mathrm{N}$ films which increases with increasing $\mathrm{N}$ content.

3. For $E>15 \mathrm{~V} / \mu \mathrm{m}\left(J>10^{-6} \mathrm{~A} / \mathrm{cm}^{2}\right)$, for ta-C films with different thicknesses, and for $E>10-12 \mathrm{~V} / \mu \mathrm{m}$ ( $J$ $>10^{-7}-10^{-6} \mathrm{~A} / \mathrm{cm}^{2}$ ) for ta-C: $\mathrm{N}$ films with different $\mathrm{N}$ contents, FN plots tend to saturate and $\log I$ vs $\log V$ curves have comparatively lesser value of slope ranging between 6 and 10 for both ta-C and ta-C: $\mathrm{N}$ films. It increases with the decrease of film thickness in ta-C films and with the increase of $\mathrm{N}$ content in ta-C: $\mathrm{N}$ films.

In general, nitrogen incorporation in DLC films leads to the following: (i) reduction of residual stress, ${ }^{32,36,37}$ (ii) re-

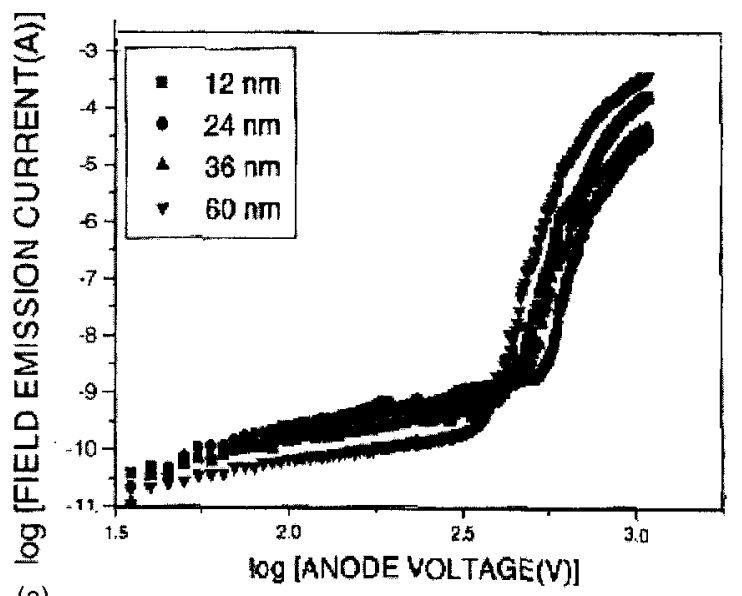

(a)

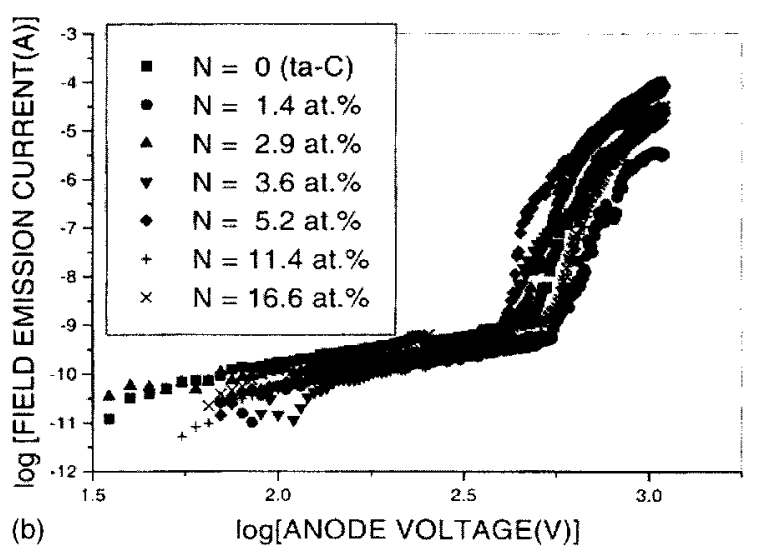

FIG. 6. (a) Variation of $\log I$ vs $\log V$ for ta-C films at different thicknesses. (b) Variation of $\log I$ vs $\log V$ for ta-C: $\mathrm{N}$ films at six different $\mathrm{N}$ contents.

duction of $E_{g},{ }^{31-33,38}$ and (iii) enhancement of conductivity. ${ }^{38}$ All these factors need to be carefully understood while discussing field emission of nitrogenated DLC. However, the universality of stress reduction on nitrogen incorporation in the $a-\mathrm{C}: \mathrm{H}$ network is well documented. ${ }^{32,36,37}$ The mechanism put forward to explain this behavior involves replacement of fourfold coordinated carbon by nitrogen atoms. As the nitrogen atoms admit a coordination number equal to three at most ( $s p^{3}$ hybridized nitrogen), the replacement of carbon atoms by nitrogen atoms in $a-\mathrm{C}: \mathrm{H}$ films necessarily implies reduction of average coordination number. This, in turn, leads to the reduction of degree of over constrain and, hence, reduction of internal residual stresses. ${ }^{37}$

Now, let us look at the overall performance of any field emission device. The contribution to field emission from a semiconductor surface is electrons from the conduction band, the valence band, and/or the surface states. The field emission depends on the following: (i) the nature of the substrate material, (ii) the film, and (iii) the surface of the film. ${ }^{39}$ This implies that the silicon substrates used maintain a supply of electrons into the DLC layers, and these electrons are then transported through the DLC layers and finally the electrons tunnel from the surface of the DLC layer into the vacuum, completing the field emission. For better performance, steps (ii) and (iii) mentioned above require the depo- 
sition parameters of the DLC films to be chosen in such a way, so that the field emission from the DLC layers can be maximized at low turn-on fields. A satisfactory field emission behavior of the DLC films can probably be attributed to the presence of both graphite and diamond carbon components and there may be an optimum abundance ratio and connectivity of the network of diamond and graphitic carbon components in the DLC films where these films may perform as better field emitters. Again, in the defect subband model of field emission advanced by Huang et al. ${ }^{40}$ it is proposed that the electrons participating in the field emission phenomenon originate from the defect site band, located below the conduction band minimum. Indeed, emissivity of diamond in many experiments has been found to increase with the increase of defect concentration. ${ }^{41}$ Ilie et al. ${ }^{42}$ while reporting the $s p^{2}$ phase nanostructure on field emission from amorphous carbons stated that the optimum size of $s p^{2}$ phase is of the order of $1 \mathrm{~nm}$. These authors further reported that the size of $s p^{2}$ phase could dominate the effect of chemical bonding, $s p^{3}$ content or conductivity on field emission. Gupta $e$ t $a l^{43}$ while reporting the electron field emission and microstructure correlation in nanocrystalline carbon thin films deposited by hot-filament chemical-vapor deposition technique using $2 \% \mathrm{CH}_{4}$ in $\mathrm{H}_{2}$ stated that along with grain size dependence, the defects spatially localized states within the band gap play a crucial role and assist in lowering the emission threshold. The optimum size for the $s p^{2}$ region was determined to be $<1 \mathrm{~nm}$ which was ascribed to be a tradeoff between the need of a small structure to provide a maximum field enhancement and the need for carrying the emission current. ${ }^{42}$ A correlation of threshold field required for emission in DLC films grown by various techniques has also been proposed in the literature ${ }^{44}$ on residual stress, $E_{g}$ and characteristic energy of band tails (Urbach energy, $E_{0}$ ) and the values of $E_{\text {turn on }}$ are found to decrease with the decrease of residual stress, $E_{g}$, and $E_{0}$ as well. Decrease of $E_{g}$ correlates to the increase of graphitic components in the films, which promote electric conduction; lowering of $E_{0}$ presumably leads to the decrease of the amount of disorder, which smoothes the hopping transport of electrons through the DLC layer in the field emission devices. Yap et al. ${ }^{45}$ while studying the effect of carbon nitride bonding structure on field emission stated that carbon nitride films with predominant tetrahedral $\mathrm{C}-\mathrm{N}$ bonds were observed to emit electrons at lower threshold field. A higher density of tetrahedral $\mathrm{C}-\mathrm{N}$ bonds reduced the threshold electric field further for electron emission. Likewise, elimination of the graphitelike $\mathrm{C}=\mathrm{N}$ bonds formed on graphite planes, aligned nearly parallel to substrate surface, can enhance electron emission. The emission seems to occur from few localized places on the film and it does not depend on uniform film properties because the emission site density is low. ${ }^{46}$ It may also be noted that despite several detailed study on ta-C related materials ${ }^{46}$ and their field emission studies ${ }^{47}$ available in the literature some pitfalls in amorphous carbon studies may still exist. ${ }^{48}$

\section{CONCLUSIONS}

Field emission measurements on the as grown (undoped) ta-C films and nitrogen doped ta-C: $\mathrm{N}$ films grown, using a pulsed filtered cathodic vacuum arc technique have been carried out. The effects of varying thickness on the field emission in undoped ta-C and varying nitrogen content in ta-C: $\mathrm{N}$ films have been studied. The value of $E_{\text {turn on }}$ in as grown ta-C films is found to increase and that of $J$ is found to decrease with the decrease of thickness up to $24 \mathrm{~nm}$ whereas the $E_{\text {turn on }}$ is found to decrease and that of $J$ is found to increase on the low level of $\mathrm{N}$ incorporation up to 5.2 at. \% in ta-C films: higher levels of nitrogen beyond $\mathrm{N}$ $\sim 5.2$ at. \% reduces it since they induce graphitization in the film as a result $E_{g}$ starts shrinking. A realistic energy band diagram of ta-C: $\mathrm{N} / n^{++} \mathrm{Si}$ heterojunction has been proposed on the basis of energy band offsets data evaluated using in situ XPS and optical spectroscopy data. Field emission data are explained using this realistic energy band diagram. The field emission results reveal that there exists a barrier to emission and the main barrier to emission is at the front surface which is the key rather than the back contact and this is related to the conduction band offset of ta-C:N $/ n^{++} \mathrm{Si}$ heterojunction. The electron field emission data are explained using FN theory and is $s p^{3} / s p^{2}$ dependent.

\section{ACKNOWLEDGMENTS}

One of the authors (O.S.P.) is grateful to the Council of Scientific and Industrial Research (CSIR), New Delhi (Government of India) for the award of Raman Research Fellowship, to the Director, National Physical Laboratory, New Delhi-1 10012 (India) for granting the leave, and to the University of Cambridge, Department of Engineering, Cambridge CB2 IPZ, England for extending the facility during the course of this work.

\footnotetext{
${ }^{1}$ A. R. Krauss, O. Aiciello, T. D. Corrigan, and R. P. H. Chang, J. Vac. Sci. Technol. B 17, 705 (1999).

${ }^{2}$ W. S. Xu, T. Zheng, and R. V. Latham, J. Phys. D 26, 1776 (1993).

${ }^{3}$ A. A. Talin, T. E. Felter, T. A. Friedmann, J. P. Sullivan, and M. P. Siegal, J. Vac. Sci. Technol. A 14, 1719 (1996).

${ }^{4}$ G. A. J. Amaratunga et al., New Diamond Front. Carbon Technol. 9, 31 (1999).

${ }^{5}$ C. Wang, A. Garcia, D. C. Ingram, and M. E. Kordesch, Electron. Lett. 27, 1459 (1991).

${ }^{6}$ A. A. Talin, L. S. Pan, K. F. McCarty, H. J. Doerr, and R. F. Bunshaw, Appl. Phys. Lett. 69, 3842 (1996).

${ }^{7}$ G. A. J. Amaratunga and S. RP. Silva, Appl. Phys. Lett. 68, 2529 (1996). ${ }^{8}$ B. S. Satyanarayana, A. Hart, W. I. Milne, and J. Robertson, Appl. Phys. Lett. 71, 1430 (1997); Diamond Relat. Mater. 7, 656 (1998).

${ }^{9}$ W. A. de Heer, A. Chatelain, and D. Ugrate, Science 270, 1179 (1995).

${ }^{10}$ M. Kuttel, O. Groening, C. Emmenegger, and L. Schlapbach, Appl. Phys. Lett. 73, 2113 (1998).

${ }^{11}$ B. F. Coll, J. E. Jaskie, J. L. Markham, E. P. Menu, A. A. Talin, and P. von Allment, MRS Symposia Proceedings No. 498 (Materials Research Society, Pittsburgh, 1998), p. 185.

${ }^{12}$ A. N. Obraztsov, A. P. Volkov, and I. Yu. Pavlovsky, JETP Lett. 68, 59 (1998).

${ }^{13}$ N. S. Xu and S. Ejaz Huq, Mater. Sci. Eng., R. 48, 47 (2005).

${ }^{14}$ B. S. Satyanarayana, Niranjana S, Shounak De, R. Bhattacharyya, and O. S. Panwar, Proceedings of the Asian Symposium on Information Displays, 2006 (unpublished), Vol. 6, p. 448.

${ }^{15}$ J. Robertson, J. Vac. Sci. Technol. B 17, 659 (1999).
} 
${ }^{16}$ A. Hart, B. S. Satyanarayana, J. Robertson, and W. I. Milne, Appl. Phys. Lett. 74, 1594 (1999); Diamond Relat. Mater. 8, 809 (1999).

${ }^{17}$ R. D. Forest, A. P. Burden, S. R. P. Silva, L. K. Cheah, and X. Shi, Appl. Phys. Lett. 73, 3784 (1998).

${ }^{18}$ J. P. Zhao, Z. Y. Chen, X. Wang, T. S. Shi, and T. Yano, Appl. Phys. Lett. 76, 191 (2000).

${ }^{19}$ J. Schaffer, J. Ristein, S. Miyazaki, and L. Ley, J. Vac. Sci. Technol. A 15, 408 (1997).

${ }^{20}$ R. H. Fowler and L. W. Nordheim, Proc. R. Soc. London, Ser. A 119, 173 (1928).

${ }^{21}$ N. L. Rupesinghe, R. J. Cole, M. Chhowalla, G. A. J. Amaratunga, and P. Weightman, Diamond Relat. Mater. 9, 1148 (2000).

${ }^{22}$ L. K. Cheah, X. Shi, E. Liu, and B. K. Tay, J. Appl. Phys. 85, 6816 (1999).

${ }^{23}$ K. C. Park et al., J. Vac. Sci. Technol. B 15, 431 (1997); Appl. Phys. Lett. 70, 1381 (1997).

${ }^{24}$ A. Weber, U. Hoffmann, and C. F. Klages, J. Vac. Sci. Technol. A 16, 919 (1998).

${ }^{25}$ F. R. Chuang, C. Y. Sun, H. F. Cheng, W. C. Wang, and I. Lin, Appl. Surf. Sci. 113/114, 259 (1997)

${ }^{26}$ K. R. Lee, K. Y. Eun, S. Lee, and D. R. Jeon, Thin Solid Films 290-291, 171 (1996).

${ }^{27}$ N. Missert, T. A. Friedmann, J. Sullivan, and R. G. Copeland, Appl. Phys. Lett. 70, 1995 (1997).

${ }^{28}$ J. Schafer, J. Ristein, and L. Ley, Diamond Relat. Mater. 6, 730 (1996).

${ }^{29}$ G. A. J. Amaratunga, D. E. Siegal, and D. R. Mckenzie, Appl. Phys. Lett. 59, 69 (1991).

${ }^{30}$ M. Chhowalla, J. Robertson, C. W. Chen, S. R. P. Silva, C. A. Davis, G. A. J. Amaratunga, and W. I. Milne, J. Appl. Phys. 81, 139 (1997).

${ }^{31}$ V. S. Veerasamy, J. Yuan, G. A. J. Amaratunga, W. I. Milne, K. W. R.
Gilkes, M. Weile, and L. M. Brown, Phys. Rev. B 48, 17954 (1993).

${ }^{32}$ C. A. Davis, D. R. Mackenzie, Y. Yin, E. Kravtchinskaia, G. A. J. Amaratunga, and V. S. Veerasamy, Philos. Mag. B 69, 1133 (1993).

${ }^{33}$ J. Robertson and C. A. Davis, Diamond Relat. Mater. 4, 441 (1995).

${ }^{34}$ R. L. Anderson, Solid-State Electron. 5, 341 (1962).

${ }^{35}$ S. M. Sze, Physics of Semiconductor Devices (Wiley, New York, 1981), p. 123.

${ }^{36}$ C. J. Trong, J. M. Siverton, J. H. Judy, and C. Chand, J. Mater. Res. 5, 2590 (1990).

${ }^{37}$ D. F. Franceschini, C. A. Achete, and F. L. Friere, Appl. Phys. Lett. 60, 3229 (1992).

${ }^{38}$ O. Amir and R. Kalish, J. Appl. Phys. 70, 4958 (1991).

${ }^{39}$ J. S. Shim, E. J. Chi, H. K. Baik, and S. M. Lee, J. Appl. Phys. 37, 440 (1998).

${ }^{40}$ Z. R. Huang, P. H. Cutler, N. M. Miskovsky, and T. E. Sullivan, Appl. Phys. Lett. 65, 2562 (1994).

${ }^{41}$ V. V. Zhimov and J. J. Hren, MRS Bull. 23, 42 (1998).

${ }^{42}$ A. Ilie, A. C. Ferrari, T. Yagi, and J. Robertson, Appl. Phys. Lett. 76, 2627 (2000).

${ }^{43}$ S. Gupta, B. L. Weiss, B. R. Weiner, and G. Morell, J. Appl. Phys. 89, 5671 (2001).

${ }^{44}$ O. S. Panwar, Sushil Kumar, S. S. Rajput, Rajnish Sharma, and R. Bhattacharyya, Vacuum 72, 183 (2004).

${ }^{45}$ Y. K. Yap, S. Kida, Y. Yada, M. Yoshimura, Y. Mori, and T. Sasaki, Diamond Relat. Mater. 9, 1228 (2000).

${ }^{46}$ J. Robertson, Mater. Sci. Eng., R. 37, 129 (2002).

${ }^{47}$ J. T. H. Tsai, K. B. K. Teo, and W. I. Milne, J. Vac. Sci. Technol. B 20, 1 (2002).

${ }^{48}$ Y. Lifshitz, Diamond Relat. Mater. 12, 130 (2003). 